\title{
De nouvelles pages pour les Carnets. Éditorial du comité de direction
}

Lucie Bony, Jean Estebanez and Fabrice Ripoll

\section{OpenEdition}

1 Journals

Electronic version

URL: http://journals.openedition.org/cdg/4200

DOI: $10.4000 /$ cdg. 4200

ISSN: 2107-7266

Publisher

UMR 245 - CESSMA

\section{Electronic reference}

Lucie Bony, Jean Estebanez and Fabrice Ripoll, « De nouvelles pages pour les Carnets. Éditorial du comité de direction », Carnets de géographes [Online], 12 | 2019, Online since 14 November 2019, connection on 24 September 2020. URL : http://journals.openedition.org/cdg/4200 ; DOI : https:// doi.org/10.4000/cdg.4200

This text was automatically generated on 24 September 2020 .

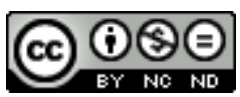

La revue Carnets de géographes est mise à disposition selon les termes de la Licence Creative Commons Attribution - Pas d'Utilisation Commerciale - Pas de Modification 4.0 International. 


\title{
De nouvelles pages pour les Carnets. Éditorial du comité de direction
}

\author{
Lucie Bony, Jean Estebanez and Fabrice Ripoll
}

1 Après neuf ans et dix numéros, les fondatrices des Carnets ont décidé de renouveler le comité de direction ${ }^{1}$. Nous avons proposé un projet au comité de rédaction qui l'a discuté et approuvé - une pratique qui n'est sans doute pas si courante. Nous inscrivant très largement dans la continuité de ce qui a été fait depuis la création de la revue, nous proposons d'accentuer et de développer plus spécifiquement les facettes suivantes.

\section{Ancrer plus fortement la géographie dans les sciences sociales}

2 Carnets de géographes est une revue qui ancre pleinement la géographie dans les sciences sociales et qui a su se montrer ouverte aux recherches dites critiques, consacrées à la dimension spatiale des inégalités et des rapports de pouvoir ou de domination. Nous pensons qu'il faut absolument maintenir cette orientation: il n'y a pas beaucoup de revues en France pour la porter, et les Carnets sont sans doute déjà identifiés comme y contribuant. Cette posture, que nous portons, ne constitue évidemment pas une norme impérative et nous continuerons à publier des articles portant d'autres types d'orientations (comme le montre ce numéro).

3 Le dialogue avec les autres sciences sociales, annoncé et désiré, nous semble pouvoir être encore davantage développé. Le nom de la revue n'aide peut-être pas à faire émerger spontanément cette ouverture, surtout dans le contexte où la géographie est dominée et encore très souvent ignorée ou disqualifiée. Toutefois, pour faire connaître la revue par-delà ses frontières, nous souhaitons renforcer les relations et collaborations avec la sociologie, l'anthropologie, la science politique, l'histoire, la psychologie, le droit, l'économie, la littérature, etc. La création du comité de correspondantes contribuera à cette dynamique (cf. infra). Nous envisageons aussi de consacrer un ou plusieurs numéros thématiques à cette réflexion sur le transdisciplinaire, pour lancer ce travail en commun. 
4 Si la réflexivité - c'est-à-dire l'analyse des activités de recherche considérées comme des pratiques sociales, et des chercheuses et chercheurs comme socialement situées est sans doute moins développée en géographie que dans d'autres disciplines, elle est fortement promue par la rubrique "Carnets de terrain». Nous souhaitons donc maintenir cette rubrique novatrice qui permet notamment d'ouvrir les cuisines de la recherche. Il s'agira à l'avenir de renforcer cette invitation au pluralisme méthodologique (en ne négligeant pas les méthodes quantitatives encore peu investiguées dans les numéros des Carnets déjà parus), à l'innovation, mais aussi à la réflexivité.

\section{Promouvoir d'autres thématiques, valoriser débats et controverses}

5 La revue s'est clairement positionnée comme accueillant des travaux et réflexions sur des thèmes peu ou pas traités ailleurs. Sans qu'il s'agisse de courir après les modes, les dossiers étant discutés collectivement, cette perspective originale nous semble importante à maintenir. Cela passe bien sûr par des numéros thématiques mais aussi par le maintien de varia, qui permettent de faire connaitre des recherches encore peu développées ou partagées.

6 Nous aimerions également accorder une place plus importante aux débats. La géographie manque peut-être un peu de culture de la controverse. Nous envisageons de concevoir ou accueillir des numéros qui abordent de front des désaccords qui semblent encore tacites ou même peu conscients. S'il existe une rubrique « Carnets de débats », il y a aussi de place pour le débat dans les "Carnets de recherches »! Enfin, la rubrique «Carnets de lectures » accueillera volontiers des notes critiques et ouvrira la possibilité de discussions avec les autrices et auteurs.

\section{Quelques nouveautés}

7 A la suite du numéro 10 consacré à l'enseignement, nous souhaitons accorder une plus grande place à cette question dans la revue. Une nouvelle rubrique «Carnets d'enseignements" est ainsi créée. Cette rubrique accueillera des travaux réflexifs d'enseignantes du primaire au supérieur, sous forme de témoignages et de retour d'expériences. L'enjeu est aussi de réfléchir aux conditions matérielles qui rendent possibles ces expériences (question de leur reproductibilité). Cette rubrique pourra aussi accueillir des entretiens, des comptes-rendus de tables-rondes sur la construction des maquettes (ESPE, LMD4), des points sur la question du recrutement des étudiantes (en licence, master...), des synthèses de mémoires de master ESPE, etc. En cohérence avec la ligne éditoriale de la revue, cette rubrique s'inscrit dans une perspective pluridisciplinaire.

Autre nouveauté: le "comité scientifique» est remplacé par un «comité de correspondant.es ». Le comité scientifique avait un rôle de garant intellectuel, crucial au moment de la fondation de la revue. Certains de ses membres ont d'ailleurs été de précieux soutiens en participant activement à la vie de la revue. Après dix numéros et presque autant d'années d'existence, cette fonction de garant n'est plus nécessaire. Les membres du comité scientifiques ont donc été invitées à rejoindre, s'ils ou elles le 
désiraient, un tout nouveau comité de correspondantes où ils ou elles pourront côtoyer d'autres collègues géographes mais aussi des chercheuse et chercheur issu'es d'autres disciplines. Ses membres sont invitées à relayer les appels à communication et les publications de numéros dans leurs réseaux et seront également de précieux soutiens dans l'évaluation des articles qui nous sont soumis de la part de non géographes. Le comité de correspondantes pourra ainsi participer, à moyen terme, à une recomposition partielle du comité de rédaction, en y intégrant des non-géographes intéressées par la dimension spatiale de nos sociétés.

\section{Des projets à développer...}

L'ouverture aux jeunes chercheuses et chercheurs et la place accordée aux «premiers écrits » est une caractéristique marquante de la revue. Nous souhaiterions davantage l'afficher, car cela se pratique peu dans le champ des revues en sciences sociales. Pour mémoire, cette pratique consiste à accompagner un auteur ou une autrice dans le travail d'écriture lui-même par de nombreux allers-retours, en soulevant, pour l'auteur ou l'autrice et l'évaluateur ou l'évaluatrice concernée, l'anonymat. Cet apprentissage à l'écriture scientifique, très inégalement accessible aux doctorants et doctorantes nous semble en effet crucial. Dans le même ordre d'idée, il s'agit de poursuivre l'enrichissement de la rubrique "Carnets de soutenances", qui offre un espace de visibilité de travaux de doctorats récemment soutenus.

10 L'ouverture de la revue à l'international est à développer. On pense d'abord au bilinguisme avec des traductions en anglais de certains au moins de nos articles : cela pourrait être un horizon à moyen-long terme mais supposerait un vaste travail et des soutiens financiers. Toutefois, la revue invite d'ores et déjà à des traductions en français de textes publiés dans d'autres langues qu'il serait intéressant de faire connaître que ce soit dans le cadre d'un dossier thématique ou d'un varia. Des entretiens avec des chercheuses et chercheurs étrangeres pourraient aussi permettre cette ouverture en aidant à passer les barrières de la langue.

11 Merci encore à Karine Ginisty, Amandine Spire et Jeanne Vivet d'avoir lancé cette aventure des Carnets et à toutes celles et ceux qui l'ont fait vivre! Merci aussi à celles et ceux qui la poursuivent à nos côtés. Nous espérons que les nouvelles pages qui s'écriront dans les années à venir seront à la hauteur des précédentes. La revue continue!

\section{NOTES}

1. Karine Ginisty, Amandine Spire et Jeanne Vivet, «Éditorial du comité de direction. Quoi de neuf après dix numéros ? ", Carnets de géographes [En ligne], 11 | 2018, mis en ligne le 15 novembre 2018. URL : http://journals.openedition.org/cdg/1683. 


\section{AUTHORS}

\section{LUCIE BONY}

Chargée de recherche au CNRS, UMR 5319 Passages

lucie.bony@cnrs.fr

\section{JEAN ESTEBANEZ}

Maître de conférences en géographie, UPEC, Lab’urba

jean.estebanez@u-pec.fr

\section{FABRICE RIPOLL}

Maître de conférences en géographie, UPEC, Lab'urba

fabrice.ripoll@u-pec.fr 